\title{
DETERMINANTES DEL CONSUMO DE PRODUCTOS ORGÁNICOS: CASO DE ESTUDIO LOJA, ECUADOR
}

\author{
Elisa Toledo Macas ${ }^{1 *}$ \\ ${ }^{1}$ Universidad Técnica Particular de Loja, Departamento de Economía, Loja - Ecuador \\ *Autor para correspondencia: e-mail: eetoledo@utpl.edu.ec \\ Recibido: 2015/27/10 Aprobado: 2018/19/05 \\ D0l: https://doi.org/10.26621/XIV18.2018.06.A06.PUCESI.2550.6684
}

\section{RESUMEN}

La presente investigación analiza la influencia de variables económicas, sociodemográficas, psicográficas y de conocimiento sobre la probabilidad de que los hogares consuman productos orgánicos en el cantón Loja, Ecuador, durante el año 2015. Los datos fueron tomados de una encuesta a 397 hogares, tanto del sector urbano como rural; con esta información se realizó la estimación de un modelo de regresión logística. Los resultados permiten concluir que únicamente las variables psicográficas, como la actitud de los hogares hacia el medio ambiente y el conocimiento sobre los beneficios de consumir este tipo de productos influyen positiva y significativamente sobre la probabilidad de consumir productos orgánicos. Es decir, si a los hogares les preocupan los problemas que afectan al medio ambiente y conocen las ventajas de consumir productos orgánicos, la probabilidad de que éstos consuman este tipo de productos va a aumentar. En contraste, las variables económicas como el ingreso y las variables sociodemográficas como el género, edad, estado civil, nivel de instrucción, número de miembros del hogar y el área de residencia no son buenas predictores del consumo de productos orgánicos. Una implicación de política, derivada de los resultados, es organizar campañas de difusión sobre los beneficios que trae consigo el consumo de productos orgánicos, tanto para la salud de las personas como para el medio ambiente; además, crear espacios para que los agricultores orgánicos den a conocer a la ciudadanía la oferta de sus productos.

Palabras clave: consumo orgánico, económicas, sociodegráficas, psicográficas, conocimiento

\begin{abstract}
The present research analyzes the influence of economic, sociodemographic, psychographic and knowledge variables on the probability that households get to consume organic products in Loja, Ecuador, during 2015. The data was taken from a survey of 397 households from both urban and rural sector; with this information, an estimation of a logistic regression model was made. The results allowed us to conclude that only psychographic variables, such as the attitude of households towards the environment and knowledge about the benefits of consuming this type of products, have a positive and significant influence on the probability of consuming organic products. That is, if households are concerned about the problems that affect the environment and know the advantages of consuming organic products, the probability that they consume these types of products will increase. In contrast, economic variables such as income, and sociodemographic variables like gender, age, marital status, level of education, number of household members and the area of residence are not good predictors of the consumption of organic products. A policy implication derived from the results is to organize dissemination campaigns about
\end{abstract}


the benefits brought by the consumption of organic products, both for health of the people and for the environment; also create spaces for organic farmers to inform the public about the offer of their products.

Keywords: organic consumption, economic, sociodegraphic, psychographic, knowledge

\section{INTRODUCCIÓN}

La creciente preocupación por el medio ambiente ha generado un cambio en los hábitos de consumo por parte de los hogares, quienes cada día buscan productos cuyo origen sea lo más natural posible; de tal forma, que garanticen que su producción no comprometa el medio ambiente y que además sea beneficioso para la salud. De manera específica, el incremento del uso de insecticidas y plaguicidas químicos utilizados en la agricultura convencional y la proliferación de productos genéticamente modificados, aumentan el riesgo percibido por los consumidores (Olivas y Bernabéu, 2012). En este sentido, los productos procedentes de la agricultura orgánica, basada en la no utilización de productos contaminantes, es la respuesta a la preocupación de los consumidores.

Los productos orgánicos son aquellos productos alimenticios (vegetales y animales) obtenidos sin el uso de sustancias químicas (pesticidas, herbicidas, fertilizantes sintéticos) ni el uso de Organismos Genéticamente Modificados, y que tienen un reconocimiento por tal manejo (Andrade - Ortiz \& Flores, 2008). En este contexto se puede concluir que, su producción supone una vuelta a la agricultura tradicional como rechazo a las prácticas de la agricultura moderna (Conford, 2001).

A finales de la década de los ochenta, la demanda por los productos orgánicos, empezó a incrementarse en los países desarrollados, según los datos más recientes de la Organización de las Naciones Unidas para la Alimentación y la Agricultura (FAO), cerca de 30 millones de hectáreas se utilizan a nivel mundial para la producción orgánica. Australia es el país con mayor superficie destinada a la producción orgánica con casi 12 millones de hectáreas, le sigue Europa, con más de 7 millones y América Latina, con casi 5 millones. En Latinoamérica, los países con mayor producción de alimentos orgánicos son Argentina, Brasil y Chile (FAO, 2017).

En el Ecuador la superficie cultivada se ha incrementado sustancialmente, llegando a ocupar el $0.69 \%$ de la superficie total cultivada en el 2011 (IFOAM, 2013). Entre los principales productos orgánicos cultivados destacan el cacao, café, banano, caña de azúcar y quinua, los cuales son destinados fundamentalmente a la exportación a países de la Unión Europea (Andrade-Ortiz \& Flores, 2008).

Bajo este contexto, se puede evidenciar que la producción orgánica aún es escasa, lo cual responde a un bajo consumo por parte de los hogares. De acuerdo a Andrade - Ortiz \& Flores (2008), solo 7 de cada 100 ecuatorianos conocen los productos orgánicos/agroecológicos y apenas 5 de cada 100 afirmaron haberlos consumido, esto como resultado de la falta de conocimiento sobre los beneficios de este tipo de productos. Esta realidad no es ajena al resto de países ya que un significativo porcentaje de la población, aún no tiene claro qué es un producto orgánico y en qué se diferencia de uno convencional. Por lo tanto, el desconocimiento es una de las principales limitantes del consumo (Williamson, 2007; Hughner, Mcdonagh, Prothero, Shultz \& Stanton,., 2007; Fuentes \& López de Coca, 2008).

La literatura desarrollada hasta la actualidad, ha identificado otras variables que influyen a la hora de elegir el consumo de productos orgánicos, la cual puede clasificarse en variables económicas, sociodemográficas y psicográficas (Díaz, Adasme-Berríos, Rodríguez \& Pindado, 2013). Dentro de las variables económicas y sociodemográficas se incluyen la edad, el sexo, el ingreso, nivel de estudios, área de residencia, entre otras; algunos estudios encuentran una relación significativa entre estas variables y la probabilidad de que los hogares consuman productos orgánicos (Van Liere y Dunlap, 1981; Garcés, Pedraja y Rivera, 1995; Fraj, Martínez \& Polo, 1999). En esta misma línea, Wier, Hansen, Andersen, \& Millock, (2003) encontraron que el mayor porcentaje de consumidores de productos orgánicos se concentra en mujeres jóvenes y con estudios superiores; y entre adultos independientes y parejas de adultos sin hijos o con hijos mayores (Dettmann \& 
Dimitri, 2007; Vega, Parras \& Torres, 2007). En contraste, otros autores encuentran que las variables como la edad, género, estado civil, nivel de ingresos no son buenas predictoras del consumo de productos orgánicos $y$, por el contrario, señalan que las variables, como el conocimiento que los consumidores poseen sobre los beneficios de los productos orgánicos es el principal determinante a la hora de elegir un producto orgánico (Brooker, 1976; Montoro, 2003; Fraj \& Martínez 2003).

Los estudios sobre el consumo de productos orgánicos, también han incluido como determinantes las variables psicográficas, las cuales, son variables internas al individuo y, por tanto, de carácter subjetivo y muy difíciles de medir (Santesmases, 2001). Estas variables incluyen aspectos relacionados con los valores y creencias de los individuos, con su estilo de vida, sus actitudes, su personalidad y otros factores psicológicos (motivación, percepción, aprendizaje, etc.) (Fraj, 2003). Entre las principales conclusiones, respecto a la relación entre las variables psicográficas y el consumo de productos orgánicos (como parte del comportamiento ecológico), tenemos que los resultados obtenidos reflejan relaciones estadísticamente significativas, es decir, las actitudes de los consumidores respecto al medio ambiente, incrementan la probabilidad de consumir productos orgánicos (De Young,1985; Dunlap \& Van Liere, 1986; Granzin \& Olsen, 1991; Fraj et al., 1999; Chan, 1999, Sánchez, 2000; Tsakiridou, Mattas, \& Tzimitra-Kalogianni,.., 2006; Williamson, 2007; Ahmad y Juhdi, 2008). En contraste autores como Hughner et al. (2007) han encontrado que la preocupación por el medio ambiente no es un factor importante para analizar el consumo de productos orgánicos.

La preocupación por los riesgos para la salud ha sido otra de las principales variables que influyen sobre la decisión de consumir productos orgánicos (Zanoli y Naspetti, 2002; Aldonado y Almansa, 2009), considerando su condición libre de pesticidas como el atributo más importante (Vega et al., 2007). Además, hay que tener en cuenta que, la decisión de consumir o no productos orgánicos también puede variar de acuerdo al país, al grado de preocupación por el medio ambiente y a la disponibilidad de estos productos (Ahmad y Juhdi, 2008). Con estos antecedentes se puede evidenciar que los estudios realizados para analizar el consumo de productos orgánicos y sus determinantes son aún escasos en países menos desarrollados, y más aún, a nivel local donde la actividad agrícola es más frecuente (Alvarado \& Toledo, 2017). El objetivo de esta investigación es analizar la influencia de las variables sociodemográficas, económicas, psicográficas y de conocimiento al momento de tomar la decisión de consumir productos orgánicos por parte de los hogares del cantón Loja. Para el análisis, se utilizó un modelo de regresión logística para una variable dependiente binaria. La importancia de conocer las variables que influyen sobre los hogares para consumir productos orgánicos radica en que, a través de los resultados, se pueden recomendar políticas locales y nacionales, orientadas a incrementar el consumo de productos orgánicos entre la población y, a la vez, mejorar los ingresos de pequeños productores.

El resto del documento está organizado de la siguiente manera. La Sección 2 presenta los datos y métodos usados dentro del estudio. La Sección 3 se enfoca en los resultados de la aplicación de la metodología y una discusión de los principales hallazgos encontrados, frente a la evidencia empírica. Finalmente, en la Sección 4 se presentan las conclusiones y recomendaciones.

\section{MATERIALES Y MÉTODOS}

\section{Datos}

La información sobre el consumo de productos orgánicos y las variables sociodemográficas, económicas, psicosociales y de conocimiento se obtuvo a través de una encuesta. El diseño de la misma se basó en el estudio "Greening Household Behaviour" de la Organización para la Cooperación y el Desarrollo Económicos (OCDE, 2011), e incluyó cuatro secciones; en la primera se abordaron preguntas relacionadas a las características sociodemográficas y económicas, en la segunda se recogió información de variables psicosociales que incluían actitudes y conductas con el medio ambiente; en la tercera sección se realizaron preguntas sobre el conocimiento del hogar en temas ambientales (educación ambiental) y el conocimiento sobre los productos orgánicos. Finalmente, en la última sección, se incluyó información sobre el consumo de productos orgánicos y los motivos por los cuales se consumen o no estos productos. 
La encuesta se aplicó a 397 hogares del cantón Loja, tanto del sector urbano como rural. El número de encuestas se determinó mediante la fórmula del tamaño de muestra para población finita, con el 95\% de confianza y un margen de error del 5\%. Para obtener el tamaño de la muestra, de cada parroquia del cantón Loja, se realizó un muestreo estratificado proporcional, en la cual la distribución se hace de acuerdo con el tamaño de la población de cada parroquia. A continuación, se presenta la definición de las variables y las estadísticas descriptivas.

Tabla 1. Estadísticas descriptivas de las variables

\begin{tabular}{|c|c|c|c|c|c|c|}
\hline Variable & Descripción & Obs & Media & $\begin{array}{l}\text { Desviación } \\
\text { estándar }\end{array}$ & Min & Max \\
\hline consumo & $\begin{array}{l}\text { Variable dicotómica Decisión del hogar de } \\
\text { consumir productos orgánicos. ( } 1=\text { si consu- } \\
\text { me; } 0=\text { no consume). }\end{array}$ & 397 & 0.62 & 0.48 & 0 & 1 \\
\hline área & $\begin{array}{l}\text { Variable dicotómica. Área de residencia del } \\
\text { Hogar. (1=urbana; } 0=\text { rural). }\end{array}$ & 397 & 0.85 & 0.35 & 0 & 1 \\
\hline género & $\begin{array}{l}\text { Variable dicotómica Sexo del Jefe de Hogar. } \\
\text { (1=Hombre; } 0=\text { =Mujer). }\end{array}$ & 397 & 0.31 & 0.46 & 0 & 1 \\
\hline estado_civil & $\begin{array}{l}\text { Variable dicotómica (1=Casado, unión libre; } \\
0=\text { otros). }\end{array}$ & 397 & 0.68 & 0.46 & 0 & 1 \\
\hline miembros_hogar & $\begin{array}{l}\text { Número de miembros del hogar(variable conti- } \\
\text { nua) }\end{array}$ & 397 & 4.11 & 1.70 & 1 & 11 \\
\hline edad & Variable continua. & 397 & 41.53 & 14.24 & 17 & 82 \\
\hline educación & $\begin{array}{l}\text { Nivel de instrucción. Variable dicótoma ( } 1=\text { ins- } \\
\text { trucción superior; } 0=\text { otros) }\end{array}$ & 397 & 0.42 & 0.49 & 0 & 1 \\
\hline ingresos & $\begin{array}{l}\text { Variable dicótoma ( } 1=\text { mayor o igual al salario } \\
\text { básico; } 0=\text { menor al salario básico }{ }^{\star}\end{array}$ & 397 & 0.39 & 0.49 & 0 & 1 \\
\hline conocimiento & $\begin{array}{l}\text { Variable dicótoma ( } 1=\text { conoce sobre los benefi- } \\
\text { cios de los productos orgánicos; } 0=\text { no conoce } \\
\text { sobre los beneficios de los productos orgánicos) }\end{array}$ & 397 & 0.73 & 0.44 & 0 & 1 \\
\hline actitud_ambiental & $\begin{array}{l}\text { Variable dicótoma }(1=\text { le preocupa la situación y } \\
\text { problemas que afectan al medio ambiente; } 0= \\
\text { no le preocupa el medio ambiente) }\end{array}$ & 397 & 0.83 & 0.37 & 0 & 1 \\
\hline
\end{tabular}

* Para el año 2015 el salario básico en Ecuador fue de 354 dólares.

\section{Estimación del modelo econométrico}

Con el propósito de determinar la probabilidad de consumir o no productos orgánicos, en función de las características sociodemográficas, económicas, psicosociales y de conocimiento de los hogares del cantón Loja, se aplicó un modelo de regresión logística binaria. El modelo LOGIT, es un procedimiento por medio del cual se intentan analizar las relaciones de asociación entre una variable dependiente dicotómica (binaria o dummy) y una o varias variables independientes (regresores o predictores). En este caso, la variable dependiente dicotómica consiste en: si consume productos orgánicos (CONSUME=1) o no consume (CONSUME=0).

La especificación empírica incluyó variables como el área, género, estado civil, número de miembros del hogar, edad, educación, ingresos, conocimiento sobre los beneficios de los productos orgánicos y actitud ambiental; las funciones se plantean de la siguiente forma:

$$
\begin{gathered}
\text { Prob }(\text { CONSUME }=1)=\mathrm{F}(\mathrm{s}, \theta) \\
\operatorname{Pr} \text { ob }(\text { CONSUME }=0)=1-\mathrm{F}(\mathrm{s}, \theta)
\end{gathered}
$$


Donde $\theta$ es el vector de los parámetros de cada función, los cuales reflejan el efecto que cada una de las variables (contenidas en el vector s respectivamente) tiene sobre la probabilidad de consumir productos orgánicos (Toledo \& Morocho, 2016). La función econométrica de las variables incluidas se especifica en la ecuación 1.

$\operatorname{Prob}($ CONSUME $=1)=\mathrm{F}\left(\alpha_{-1}+\theta_{-1}\right.$ área $+\theta_{-2}$ género $+\theta_{-3}$ estado_civil $+\theta_{-4}$ miembros $_{- \text {hogar }}+\theta_{-5}$ edad $+\theta_{-6}$ educación $+\theta_{-7}$ ingresos $+\theta_{-8}$ conocimiento $+\theta_{-9}$ actitud)

Además, con la finalidad de interpretar el efecto de cada una de las variables independientes sobre la probabilidad de éxito de la variable dependiente, se utilizan los efectos marginales del modelo LOGIT, el cual se expresa en la siguiente forma funcional:

$$
\frac{\partial E[\operatorname{CONSUME} \mid s]}{\partial s}=\left\{\frac{d F\left(\theta^{\prime} s\right)}{d\left(\theta^{\prime} s\right)}\right\} \theta=f\left(\theta^{\prime} s\right) \theta
$$

\section{RESULTADOS Y DISCUSIÓN}

La Tabla 2 reporta los resultados que se obtuvieron de la estimación de la función econométrica formalizada en la ecuación 1 en la sección anterior.

Tabla 2. Resultados modelo LOGIT consumo de productos orgánicos

\begin{tabular}{|c|c|}
\hline & [consumo_orgánicos] \\
\hline \multirow[t]{2}{*}{ área } & 0.510 \\
\hline & $(1.60)$ \\
\hline \multirow[t]{2}{*}{ género } & 0.0375 \\
\hline & $(0.15)$ \\
\hline \multirow[t]{2}{*}{ estado_civil } & -0.0465 \\
\hline & $(-0.18)$ \\
\hline \multirow[t]{2}{*}{ edad } & 0.00486 \\
\hline & $(0.53)$ \\
\hline \multirow[t]{2}{*}{ miembros_hogar } & 0.129 \\
\hline & $(1.66)$ \\
\hline \multirow[t]{2}{*}{ educación } & 0.406 \\
\hline & $(1.63)$ \\
\hline \multirow[t]{2}{*}{ ingresos } & 0.232 \\
\hline & $(0.90)$ \\
\hline \multirow[t]{2}{*}{ conocimiento_prod_org } & $1.301^{* \star *}$ \\
\hline & $(5.05)$ \\
\hline \multirow[t]{2}{*}{ actitud } & $0.934^{* *}$ \\
\hline & (3.08) \\
\hline \multirow[t]{2}{*}{ Constant } & $-2.328^{* * *}$ \\
\hline & $(-3.95)$ \\
\hline Observations & 397 \\
\hline Adjusted $R^{2}$ & \\
\hline
\end{tabular}

t statistics in parentheses ${ }^{*} p<0.05,{ }^{* *} p<0.01,{ }^{* * *} p<0.001$ 
A partir de los signos de los coeficientes estimados, se puede deducir la relación entre las variables independientes y la probabilidad de consumir productos orgánicos. Variables como el área de residencia, género, edad, número de miembros del hogar, nivel de educación, ingresos, conocimiento sobre los beneficios de consumir productos orgánicos y la actitud de compromiso con el medio ambiente, influyen positivamente sobre la probabilidad de consumir productos orgánicos.

Es decir, si los hogares están ubicados en el área urbana, el jefe de hogar es de sexo femenino, con instrucción superior, gana más del salario básico, conoce sobre los beneficios de consumir productos orgánicos y tiene una actitud de compromiso con el medio ambiente, la probabilidad de que se consuman productos orgánicos se va a incrementar. Así mismo, a medida que aumenta el número de integrantes del hogar, y la edad del jefe de hogar la probabilidad de consumir productos orgánicos también aumenta.

En contraste con estos resultados, la variable estado civil influyen negativamente sobre la probabilidad de consumir productos orgánicos, esto significa que el hecho de tener un estado civil "casado" disminuirá la probabilidad de consumir productos orgánicos.

Es importante mencionar que, desde el punto de vista estadístico, únicamente son significativas las variables de conocimiento sobre el beneficio de consumir productos orgánicos y actitud pro ambiente, estos resultados concuerdan con los obtenidos por De Young, (1985); Dunlap y Van Liere, (1986); Granzin et al., (1991); Fraj, (1999); Chan, (2001); Sánchez, (2000); Tsakiridou et al., (2006); Williamson, (2007); Ahmad y Juhdi, (2008); quienes encontraron relaciones muy significativas entre las variables psicográficas y de conocimiento y el comportamiento medioambiental, medido a través del consumo de productos orgánicos. En este mismo sentido, autores como Bhate y Lawler, (1997); Fraj y Martínez, (2003) señalan que existen relaciones poco significativas entre la edad, el ingreso, el sexo y el nivel de estudios y el comportamiento ambiental de los consumidores.

\section{Efectos marginales}

Con la finalidad de conocer el efecto de cada una de las variables independientes sobre la variable dependiente, se procedió a estimar los efectos marginales, los cuales indican las variaciones en la probabilidad de consumir productos orgánicos frente a un cambio de las variables sociodemográficas, económicas, psicosociales y de conocimiento. A continuación, se presentan los resultados.

De acuerdo a los resultados de la tabla anterior, se puede observar que la probabilidad de que los hogares consuman alimentos orgánicos aumenta en 30\% cuando éstos tienen conocimiento sobre los beneficios de consumir este tipo de productos, resultados que concuerdan con los hallazgos de Williamson, (2007); Hughner et al., (2007); Fuentes y López de Coca, (2008). En algunos estudios, la variable conocimiento se ha utilizado como una variable moderadora entre las variables psicográficas y el comportamiento ecológico (Ramsey y Rickson, 1976; Arbuthnot y Lingg, 1975; Grunert y Kristensen, 1992) de ahí que, es lógico pensar que la variable actitud también tiene una relación significativa con la probabilidad de consumir productos orgánicos. Los resultados reportados en la tabla 3, permiten concluir que los hogares que tengan una actitud positiva o favorable hacia el medio ambiente incrementarán su probabilidad de consumir productos orgánicos en 22\%, estos resultados son similares a los resultados encontrados por De Young,1985; Dunlap y Van Liere, 1986; Granzin et al., 1991; Fraj et al., 1999; Chan, 1999, Sánchez, 2000; Tsakiridou et al., 2006; Williamson, 2007; Ahmad y Juhdi, 2008).

De acuerdo al signo de los coeficientes, se espera que un hogar cuyo jefe con conocimiento de los beneficios de consumir productos orgánicos y que le preocupe la situación del medio ambiente, tenga una mayor probabilidad de consumir alimentos orgánicos que un jefe de hogar que no posea estas características. 
Tabla 3. Efectos marginales.

$\begin{array}{lc} & \text { [consumo_orgánicos] } \\ \text { área } & 0.1217 \\ & (1.56) \\ \text { género } & 0.0085 \\ & (0.15) \\ \text { estado_civil } & -0.0106 \\ & (-0.18) \\ \text { edad } & 0.0011 \\ & (0.53) \\ \text { miembros_hogar } & 0.0297 \\ & (1.66) \\ \text { educación } & 0.0921 \\ & (1.65) \\ \text { ingresos } & 0.0530 \\ \text { Odjusted R }{ }^{2} & (0.91) \\ \text { conocimiento_prod_org } & 0.3088^{* * *} \\ & (5.19) \\ & 0.2253^{* *} \\ & (3.06) \\ & 397 \\ & \\ & \end{array}$

\section{CONCLUSIONES}

La presente investigación se planteó con la finalidad de determinar la influencia de las variables sociodemográficas, económicas, psicográficas y de conocimiento en la probabilidad de que los hogares consuman productos orgánicos. De acuerdo a los resultados de la aplicación del modelo econométrico, se obtuvo que únicamente las variables psicográficas, representada por las actitudes de preocupación hacia el medio ambiente y el conocimiento sobre los beneficios de consumir productos orgánicos son estadísticamente significativas; además los signos de los coeficientes calculados son positivos, lo cual indica que ante un incremento de estas variables, la probabilidad de consumir productos orgánicos también se incrementará. En contraste, las variables sociodemográficas y económicas no determinan o no son buenas predictoras del consumo de productos orgánicos.

A partir de los resultados reportados, se tienen pautas afines con variables que influyen directamente en el consumo de alimentos orgánicos. Estas directrices, deben ser consideradas por los encargados de política y además por las empresas con el objetivo de desarrollar un mercado orgánico local y posteriormente uno nacional. Una de las herramientas que podría impulsar la demanda por productos orgánicos es la difusión de sus beneficios al consumirlos, beneficios tanto para la salud como para el medio ambiente.

A nivel local, se sugiere dar continuidad al programa "Feria Agroecológica y Orgánica" Ilevada a cabo por el Gobierno Autónomo Descentralizado de Loja, que se desarrolla el último domingo de cada mes en las parroquias de El Valle y Vilcabamba, con la finalidad de que las personas dedicadas a la agricultura 
orgánica puedan promocionar y vender sus productos de una manera directa a los consumidores e impulsar la venta y consumo de productos orgánicos.

\section{REFERENCIAS BIBLIOGRÁFICAS}

Ahmad, S. \& Juhdi, N. 2008. Consumer's perception and purchase intentions towards organic food products: exploring the attitude among Malaysian consumers. Annual Conference on PBFEAM. 15pp

Aldonado, A.M., \& Almansa, C. 2009. The private provision of public environment: Consumer preferences for organic production systems. Land Use Policy. (26), 669-682.

Alvarado, R., \& Toledo, E. (2017). Environmental degradation and economic growth: evidence for a developing country. Environment, Development and Sustainability, 19(4), 1205-1218.

Andrade - Ortiz, D., \& Flores, M. (2008). Consumo de productos orgánicos/agroecológicos en los hogares ecuatorianos. VECO, Quito, Ecuador.

Arbuthnot, J. \& Lingg, S. (1975). "A Comparison of French and American Environmental Behaviours, Knowledge and Attitudes", International Journal of Psychology, Vol, 10 (4), pp. 275-281.

Brooker, G. (1976). "The Self-Actualizing Socially Conscious Consumer", Journal of Consumer Research,(3), pp. 107-112.

Buttel F. \& Flinn W. (1974) Structure of support for environmental movement, 1968-1970. Rural Sociology. 39 (1): $56-69$

Chan, R. Y. (1999). Environmental attitudes and behavior of consumers in China: survey findings and implications. Journal of International Consumer Marketing, 11(4), 25-52.

Conford, P. (2001): "The origin of the organic movement". Floris Books, Edinburgh (Scotland).

Daniere, A. \& Takahashi, L. (1999). "Environmental Behaviour in Bangkok, Thailand: A Portrait of Attitudes, Values and Behaviour", Economic Development and Cultural Change, pp. 525-557.

De Young, R. (1985). Encouraging environmentally appropriate behavior: The role of intrinsic motivation.

Dettmann, R., \& Dimitri, C. 2007. Organics consumers: A demographic Portrayal of organic vegetable consumption within the United State. EAAE "International Marketing and International Trade of Quality Food Products". Bologna, Italy. 14pp.

Díaz Tobar, B., Adasme-Berríos, C., Rodríguez, M., \& Pindado, E. (2013). Variables que influyen en el consumo de alimentos orgánicos en la Región del Maule. Chile. Economia Agraria, 17.

Dunlap, R. E., \& LIERE, K. D. (1984). Commitment to the dominant social paradigm and concern for environmental quality. Social science quarterly, 65(4), 1013.

Fraj, E. (2003). Influencia de las características psicográficas y de conocimiento en el comportamiento del consumidor ecológico. Una aplicación empírica. Consejo Económico y Social de Aragón. Zaragoza.

Fraj, E. (2004). Influencia de las características psicográficas y de conocimiento en el comportamiento del consumidor ecológico. Una aplicación empírica. Master's thesis, Universidad de Zaragoza.

Fraj, E. \& Martínez, E. (2003). "Influencia de las características demográficas y socioeconómicas de los consumidores en la compra de productos ecológicos", Estudios sobre Consumo, No. 65, pp. 9-20.

Fraj, E., Martínez, E. \& Polo, Y. (1999). "Determinants of Consumer's Ecological Behaviour", Proceedings of the XXIV Annual Colloquium of the International Association for Research in Economic Psychology (IAREP), pp. 994-1005, Belgirate (Italia).

Fuentes, C., \& López de Coca, E. 2008. El consumo de productos ecológicos. Distribución y Consumo. 99:524.

Garcés, C., Pedraja, M. \& Rivera, P. (1995). "Variables sociodemográficas determinantes del comportamiento ecológico de los españoles", Actas del VII Encuentro de Profesores de Marketing. Barcelona.

Granzin, K. L., \& Olsen, J. E. (1991). Characterizing participants in activities protecting the environment: A focus on donating, recycling, \& conservation behaviors. Journal of Public Policy \& Marketing, 1-27.

Grunert, S. (1992). Everybody seems concerned about the environment, but is this concern reflected in (Danish) consumers' food choice?. Institut for Informations beh\&ling

Grunert, S.C. y Kristensen, K. (1992). "The Green Consumer: Some Danish Evidence", XXI Annual Conference of The European Marketing Academy Proceedings, Aarhus, p. 26-29.

Hughner, R., Mcdonagh, P., Prothero, A., Shultz li, C., \& Stanton, J. (2007). Who are organic food consumers? 
A compilation \& review of why people purchase organic food. Journal of Consumer Behaviour (6),p. 94-110.

International Federation of Organic Agriculture Movements- IFOAM (2013). The World of Organic Agriculture. Statistics and Emerging Trends.

Jackson, J. (1983). Measuring the dem\& for environmental quality with survey data. The Journal of Politics, 45(02), 335-350.

Montoro , F. (2003). La creación de imagen de marca mediante la utilización de atributos ecológicos. Una aproximación empirica. Tesis doctoral, Universidad de Granada.

Neuman, K. (1986). Personal values and commitment to energy conservation. Environment \& Behavior, 18(1), 53-74.

Ramsey, C. \& Rickson, R. (1976). "Environmental Knowledge and Attitudes", Journal of Environmental Education, Vol. 8, pp. 10-18.

Sánchez, M., Gil, J.M., \& Gracia, A. 2000. Segmentación del consumidor respecto al alimento ecológico: diferencias interregionales. Revista de Estudios Regionales (56): 171-188.

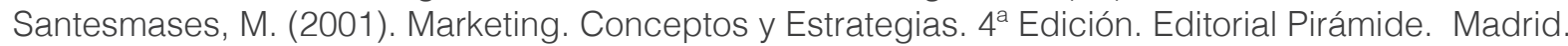

Toledo, E.., \& Morocho, T. (2016). Comportamiento Ecológico Responsable de los hogares del cantón Loja: un análisis a partir de la clasificación de los residuos sólidos. TZHOECOEN, 8(1).

Tsakiridou, E., Mattas, K., \& Tzimitra-Kalogianni, I. (2006). The influence of consumer characteristics and attitudes on the demand for organic olive oil. Journal of International Food and Agribusiness Marketing, 18(3-4), 23-31.

Van Liere, K. \& Dunlap, R. (1981). "Environmental Concern. Does it Make a Difference , How It's Measured?", Environment and Behaviour, Vol. 13 (6), pp. 651-676.

Vega, M., Parras, M., \& Torres, F.J. 2007. El comportamiento del consumidor de alimentos ecológicos en España. Un estudio exploratorio a partir de variables sociodemográficas y económicas. Conocimiento, innovación y emprendedores: camino al futuro. Universidad de Jaén: 2414-2426.

Wier, M., Hansen, L. G., Andersen, L. M., \& Millock, K. 2003. Consumer preferences for organic foods. Organic agriculture: Sustainability, markets and policies. 257pp.

Williamson, C. 2007. Facts behind the headlines. Is organic food better for our health? British Nutrition Foundation. London, UK. Nutrition bulletin. (32):104-108.

Zanoli, R., \& Naspetti, S. 2002. Consumer motivations in the purchase of organic food: a means-end approach. British Food Journal. (104)(8); 643-653. 